\title{
The Effects of an In-Season Resitance Program on Starters and Non- Starters in Elite Male Volleyball Players
}

\author{
Mário C. Marques ${ }^{1,2, *}$, Mafalda Reis ${ }^{1,2}$, Aldo M. Costa ${ }^{1,2}$, Ricardo Ferraz ${ }^{1}$, \\ Juan J. González-Badillo ${ }^{3}$ and Daniel A. Marinho ${ }^{1,2}$ \\ ${ }^{I}$ University of Beira Interior. Sport Sciences Department (UBI, Covilhã, Portugal) \\ ${ }^{2}$ Research Centre in Sports, Health and Human Development (CIDESD, Vila Real, Portugal) \\ ${ }^{3}$ University Pablo de Olavide. Sport and Informatics Department (Seville, Spain)
}

\begin{abstract}
The aim of this study was to investigate the changes in physical parameters produced during an in-season resistance training (RT) program and detraining phase in 12 top TVP competing in the elite Portuguese league. Moreover, differences in the adaptive responses between players who played most of the times (starters: S) and players that were mainly used as substitutes (non starters: NS) were analysed. This study indicated the absence of interactions for group (S or NS) by time effects for any of the tests, except for bench press. Here, S demonstrated superior upper body strength values after 12 weeks $(\mathrm{p}=0.025)$, indicating that both groups responded similarly to the training program. However, there were several main effects for time, showing significant improvements for the entire group of population. In conclusion, while no differences appear to exist in performance between starters and non-starters, it was shown that most performance variables for ST and NS can be improved with a comprehensive strength and conditioning program for TVP.

Methods: Twelve elite healthy male TVP voluntarily participated to the study. We utilized a top Volleyball squad from Portuguese I National elite division who were as a team ranked in the top 5. We monitored the squad throughout a 12week competitive in-season consisting of 14 games using a longitudinal study design. Apart from normal technical/tactical practice sessions (2-3 hours per day) and weekend competitions, all underwent 12 weeks of RT program. Upper and lower body maximal dynamic strength, jump and ball throw distance were tested at three intervals: before the experimental period (T1) and after the 12-week experimental period (T12).

Results: Of the grate interest of this research was the lack of any interactions for group (S or NS) by time effects for any of the tests, except for bench press. Here, $S$ demonstrated superior upper body strength values after 12 weeks $(\mathrm{p}=0.025)$, indicating that both groups responded similarly to the training program. However, there were several main effects for time, showing significant improvements for the entire group of population.

Discussion: The potentially greater competitive stress placed on the $\mathrm{S}$, and the differential physiological and performance effects related to S or NS status have not been clarified in prior sports research, especially in regard to volleyball players [3]. The present study found opposite results compared to those observed by Fry et al. [3]. It is difficult to compare the results because they differ markedly in a number of factors, including gender, participant's experience, and player's level. Another factor that could possibly contribute to the different outcomes between previous investigations (present study and Fry et al. [3]) with respect to the relationships between testing periods (off season versus in-season). The combination of a RT and volleyball training resulted in larger improvements in both groups. Thus, while no differences appear to exist in performance between starters and non-starters, it was shown that most performance variables for ST and NST can be improved with a comprehensive strength and conditioning program for top volleyball players.
\end{abstract}

Keywords: Strength, jumping, team volleyball, starters, non starters.

\section{INTRODUCTION}

Team Volleyball, like several other ball games, requires not only technical and tactical skills but also great deal of

*Address correspondence to this author at the Mário A.Cardoso Marques. Department of Sport Sciences, University of Beira Interior -Portugal Rua MarquêsD’ÁvilaeBolama6200001Covilhã, Portugal;

Tel: +(351) 275329153; Fax: +(351)275329157;

E-mail: mariomarques@mariomarques.com physical fitness [1, 2]. During a long competitive season typical of any European league, Team Volleyball coaches concentrate mainly on technical and tactical drills reducing the volume of training devoted to strength and conditioning activities. This may lead therefore to unwanted changes in selected aspects of the optimal physical fitness profile. To date, few studies have examined how these parameters change during a competitive season in elite Team Volleyball players (TVP). 
The aim of this study was to investigate the changes in physical parameters produced during an in-season resistance training (RT) program and detraining phase in 12 top TVP competing in the elite Portuguese league. Moreover, differences in the adaptive responses between players who played most of the times (starters: S) and players that were mainly used as substitutes (non starters: NS) were analysed. To our knowledge, only one study [3] had similar goals, however, the experiment was conduced during the off season and in female collegiate players.

\section{REFERENCES}

[1] Marques MC, González-Badillo JJ, Kluka D. In-season strength training male professional volleyball athletes. Strength Cond J 2006; 28(6): 2-12.

[2] Marques MC, Tillaar R, Vescovi J, González-Badillo JJ. Changes in strength and power performance in elite senior female professional volleyball players during the in-season: a case study. J Strength Cond Res 2008; 20(3): 563-571.

[3] Fry AC, Kraemer WJ, Waseman CA, et al. The effects of an offseason strength and conditioning program on starters and nonstarters in women's intercollegiate volleyball. J Appl Sport Sci Res 1991; 5(4): 174-81

(C) Costa et al.; Licensee Bentham Open.

This is an open access article licensed under the terms of the Creative Commons Attribution Non-Commercial License (http://creativecommons.org/licenses/by-nc/3.0/) which permits unrestricted, non-commercial use, distribution and reproduction in any medium, provided the work is properly cited. 